\title{
Structure Optimization of Microbial Fuel Cell Reactor Based on Reverse Engineering and Rapid Prototyping
}

\author{
Shuaishuai $\mathrm{Lv}^{1,2}$, Yangyang Zhu ${ }^{1,2}$, Hai Gu${ }^{2}$, Hongjun $\mathrm{Ni}^{1,2}$,Yue Meng ${ }^{1,2}$ and Xingxing Wang ${ }^{1,2}$ \\ ${ }^{1}$ School of Mechanical Engineering, Nantong University, Nantong Jiangsu 226019, China \\ ${ }^{2}$ Jiangsu Key Laboratory of 3D Printing Equipment and Application Technology, Nantong Institute of Technology, Nantong, Jiangsu 226002, \\ PR China,
}

\begin{abstract}
The structure of Microbial Fuel Cell (MFC) reactor was analyzed and improved by Reverse Engineering (RE) and Rapid Prototyping (RP) technology. Points cloud data of MFC reactor was accessed by hand-held laser scanner. The space surface and entity model were reconstructed accurately, and the structure of the reactor was optimized based on Imageware and Solidworks software. The reactor model was manufactured by RP machine. The optimization efficiency of MFC reactor was improved based on the combination of RE and RP, which has a good reference value for the development of MFC technology and products.
\end{abstract}

\section{Introduction}

Microbial fuel cells (MFCs) have emerged as a sustainable technology for wastewater treatment that has potential in recycle bioelectricity from livestock wastewater, which use microbes as catalysts instead of expensive chemical catalysts compared to conventional fuel cells (FC) [1]. However, the current power generation of MFC has not reached the actual application level, and most of the researchs on MFC are also carried out in the laboratory. The performance of MFC is affected by the structure of the reactor to a great extent and optimizing the reactor structure is one of the most important ways to improve the performance of MFC [2]. With the rapid development of new energy vehicles, update cycle of FCs is shortened, and the complicated space structure of MFC still needs to be perfected at present, of which the production engineering can not meet the requirements of its development.

Reverse engineering (RE) is a redesign process to parameterize the known objects and reconstruct the model accurately $[3,4]$. RE pays more attention to the optimization redesign process than the imitation engineering. Rapid prototyping (RP) technology is the rapid model design and manufacturing technology based on digital technology. The combination of RE and RP can greatly improve the efficiency of product optimization [5].

In this study, RE and RP were utilized to optimize the MFC reactor, of which points cloud data was gained by hand-held laser scanner and processed by Imageware software. 3D model was created and optimized by Solidworks and printed by RP machine.

\section{Parameterized measurement}

Parametric measurement is to collect the coordinate points cloud data of the object space structure by means of professional measuring equipment to parameterize the object surface. Parametric measurement includes contact measurement and non-contact measurement, in which contact measurement is of high accuracy and adaptability but time consuming and easy to destroy the model, while non-contact measurement is not accurate but efficient. According to the space curved structure characteristics of the double-chambered MFC reactor (Figure 1), the EXASCAN $^{\mathrm{TM}}$ hand-held laser scanner of Creaform company was selected [6]. Before the scanning, the imaging agent was sprayed on the surface of the bottle body and the fluorescent mark points were added according to the reactor structure (as shown in Figure 2). The destination fileof scanning was saved as .stl format after initial treatment. 


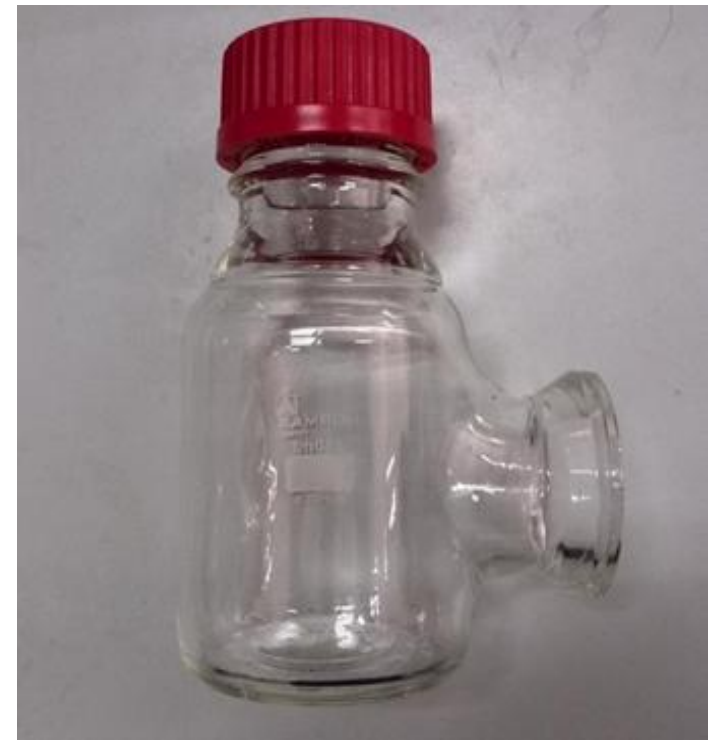

Figure 1. MFC reactor

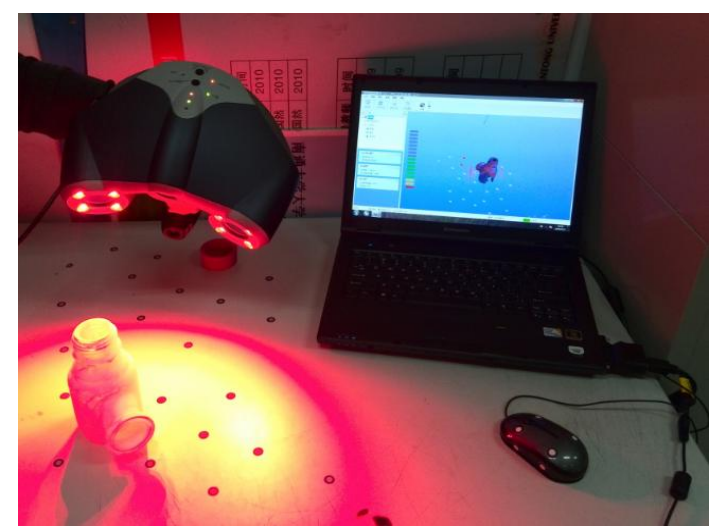

Figure 2. The scanning of MFC reactor

\section{Parametric design}

Parametric design is to reprocess the parameterized measurement data based on the general idea of point-curve-surface-entity, including pre-processing of points cloud, reconstruction of surface model and 3D solid modeling. Firstly, the redundant points generated in the scanning process were removed, and the number of the original points cloud was reduced from 16202 to 6237 using range sampling method. Based on the step by step localization method, the points cloud data of the bottle body was modified in sequence according to the characteristic relation, as shown in Figure 3.

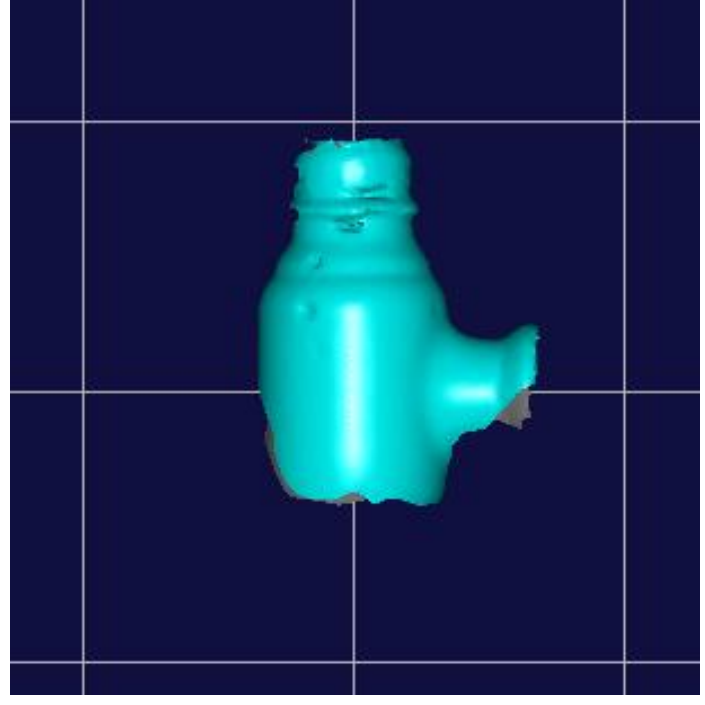

Figure 3. Position correction of points cloud data

Surface reconstruction is the process of fitting the surface based on the regularities of points cloud distribution according to the specific algorithm, in which the characteristic curve is the basis of regular surface modeling, and the characteristic curve can be used to make up the defects of the solid parts according to the design requirements to optimize product. According to the characteristic points cloud distribution fitting Non-Uniform Rational B-Splines (NURBS) surface, which is the most widely used technology of complex surface modeling, and improving the quality of NURBS surface effectively to meet the design requirements withthe modification of the control vertex. The points cloud data of the reactor bottle body was divided into four parts: the bottle body, the bottle interface, the bottleneck and the transition surface. Firstly, the points cloud of bottle body was selected and the characteristic points cloud was intercepted (Figure 4a), then the tolerance curve was constructed by points cloud and swept into the surface, and trimmed according to the actual model, as shown in Figure 4b. The processing method of the bottle interface and the bottleneck surface was the same as the bottle body's. The deviation in fitting surface was accurately adjusted with the aid of NURBS surface because the structure data of the transition surface of three rotating surfaces was complex, and the surfaces were seamlessly connected (Figure 5). Error distribution between final reconstructed surface and points cloud was analyzed as shown in Table 1., and the maximum surface deviation was $0.2734 \mathrm{~mm}$.
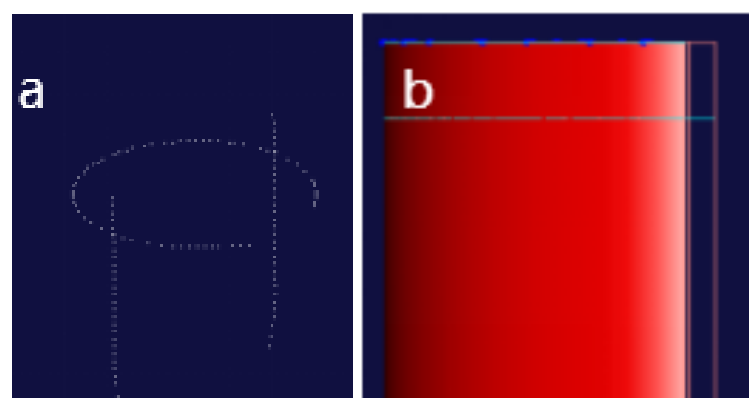

Figure 4. Surface reconstruction of bottle body 
a. Interception of characteristic points cloud; b. Trimming of swept surface

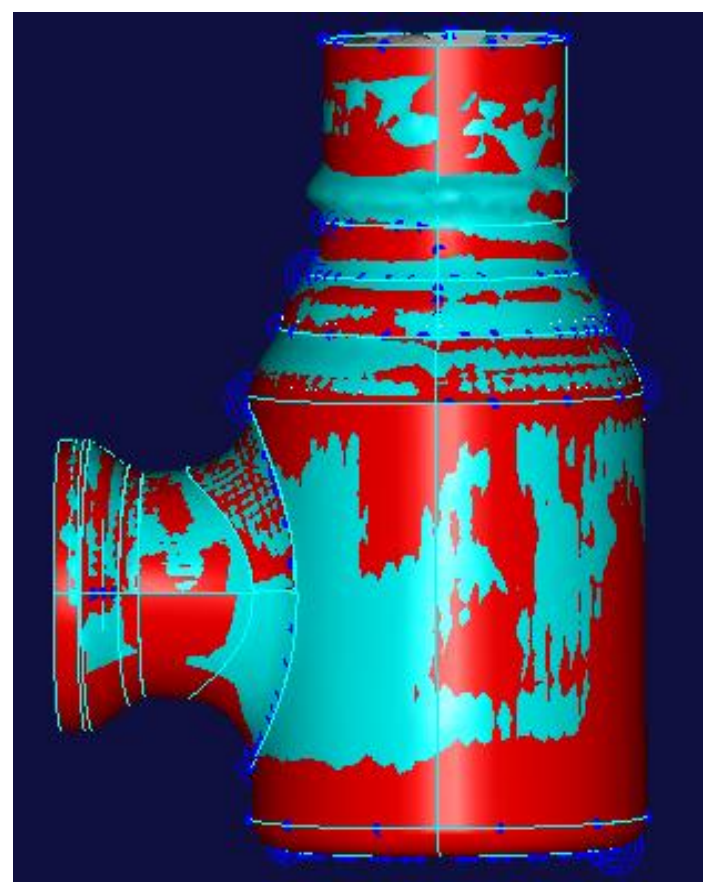

Figure 5. Reactor surface

Table 1. Error distribution between reconstructed surface and points cloud

\begin{tabular}{cccc}
\hline Deviation & $\begin{array}{c}\text { Maximal } \\
\text { Value } / \mathrm{mm}\end{array}$ & $\begin{array}{c}\text { Average } \\
\text { Value } / \mathrm{mm}\end{array}$ & $\begin{array}{c}\text { Standard } \\
\text { Value } / \mathrm{mm}\end{array}$ \\
\hline $\begin{array}{c}\text { Lateral deviation } \\
\text { Negative normal } \\
\text { deviation }\end{array}$ & 0.0000 & 0.0000 & 0.0000 \\
$\begin{array}{c}\text { Geometric } \\
\text { deviation }\end{array}$ & -0.2734 & -0.0564 & 0.0475 \\
Normal deviation & 0.12734 & 0.0531 & 0.0460 \\
\hline
\end{tabular}

The processed surface data was imported into Solidworks for 3D solid modeling, and the model was substantiated and extracted (Figure 6). The points cloud data of interface groove structure and bottleneck screw structure was not a precise measurement because of their small complex structure, which needs the utilization of Solidworks to further improve the model according to the actual measurement or design requirements (Figure 7). Because the bottle cap was complex and not of imposing stature, the 3D model was constructed directly with the aid of Soldiworks according to the design requirements (Figure 8). The final assembly diagram of the reactor was shown as Figure 9.

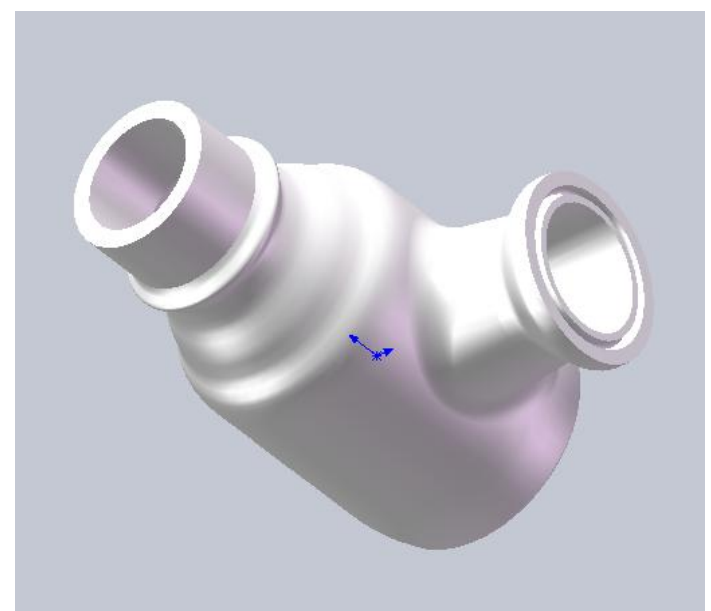

Figure 6. Reactor shell extraction

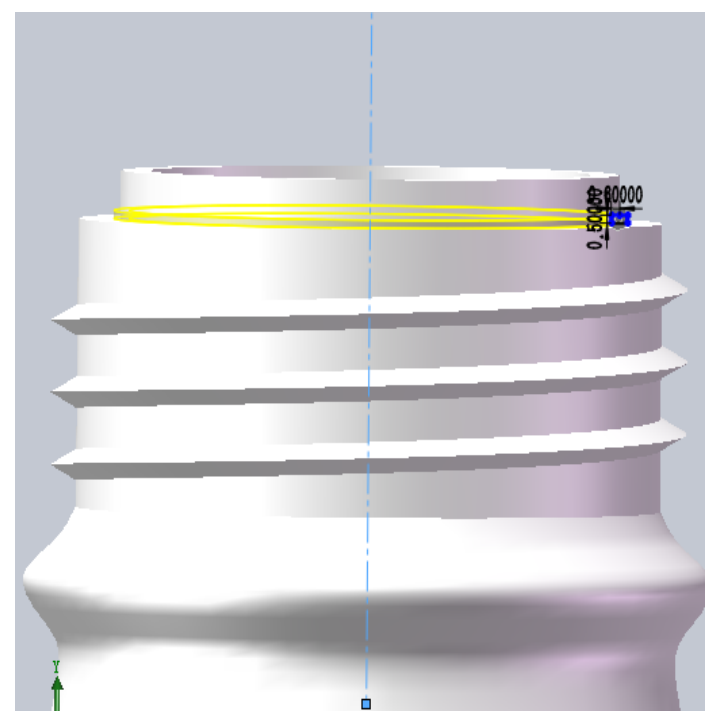

Figure 7. Sketch diagram of thread machining

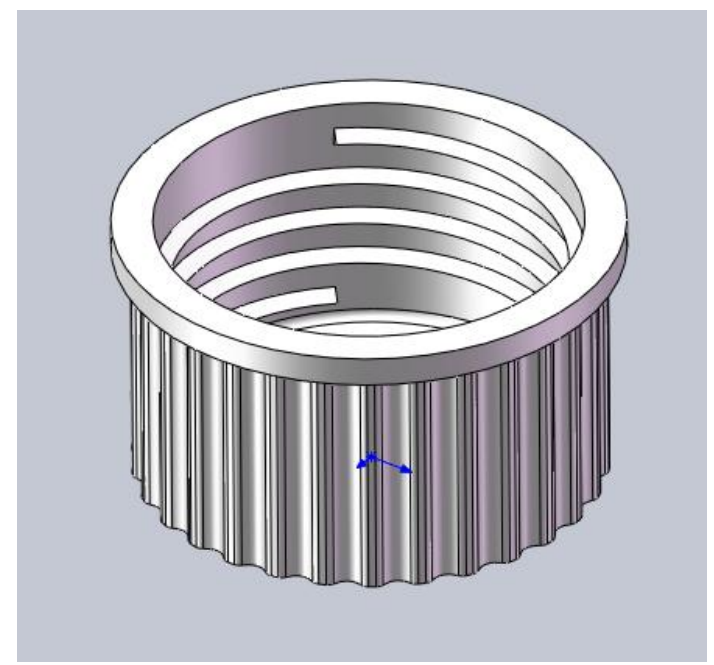

Figure 8. Bottle cap of reactor 


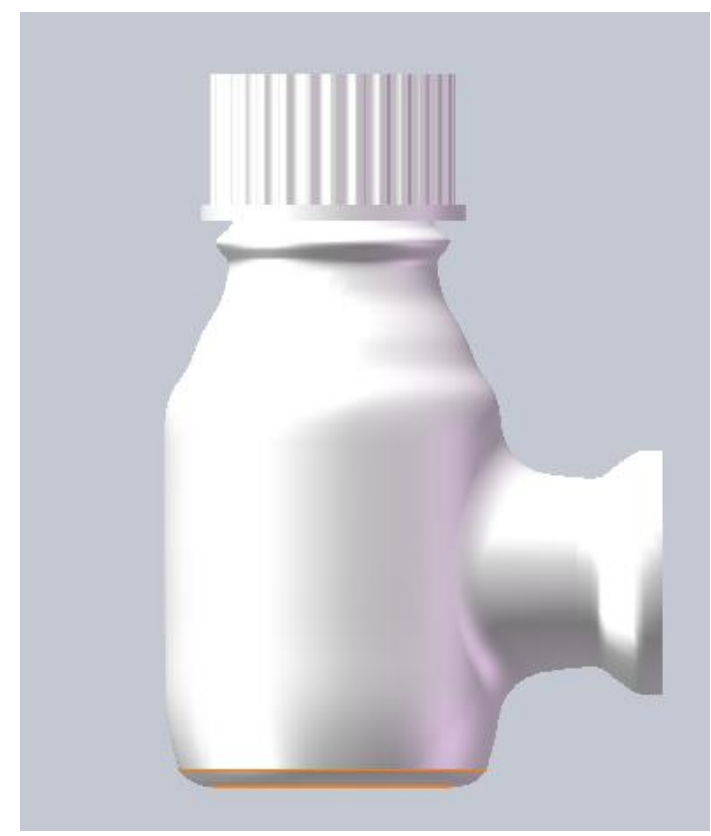

Figure 9. Reactor assembly drawing

According to the experimental operation, the phenomenon of water leakage was easy to occur around the bottle interface part of the original reactor when connected. In order to eliminate the influence of the experimental operation error on the experimental results, the groove structure of the original interface was changed into the flange shape and fixed with the threaded connection to optimize the structure of the reactor [7].

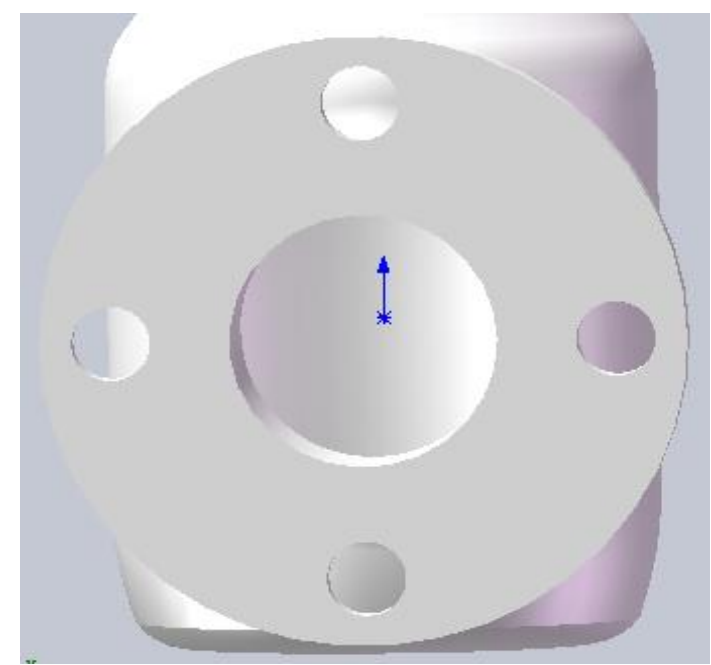

Figure 10. Flange plate of reactor

\section{Rapid Prototyping}

At present, the RP technology is to layer 3D solid model using 2D slicing method, by which obtained the section contour of each layer, in order to achieve the increase in material plane along the direction of material increase manufacturing technology [8-10]. In this study, the 3D modeling data of model was imported into the MEM320A melt extrusion rapid prototype, and the solid manufacturing of the MFC reactor was carried out based on ABS S301 and ABS B601 materials.

Entity processes for MFC reactor are as follows:

(1) Preheating. Before the RP manufacturing, preheating treatment was needed to ensure that the temperature of the molding chamber reached $60^{\circ} \mathrm{C}$, the molding material reached $260^{\circ} \mathrm{C}$ and the supporting material reached $240^{\circ} \mathrm{C}$.

(2) System initialization. Initializing the system and ensuring the safety of equipment connection.

(3) Model loading. Converting 3D model data into .stl format and importing into software, then coordinating appropriate coordinate system.

(4) Debugging. Detection and correction of model errors.

(5) Table adjustment: Setting the height of the table and the distance between the nozzle and the worktable to improve the printing efficiency.

(6) Parameter setting: Adjusting printing parameters, such as precision, support material, etc.

(7) Start printing: The machine starts automatically and stopping after the model print was finished.

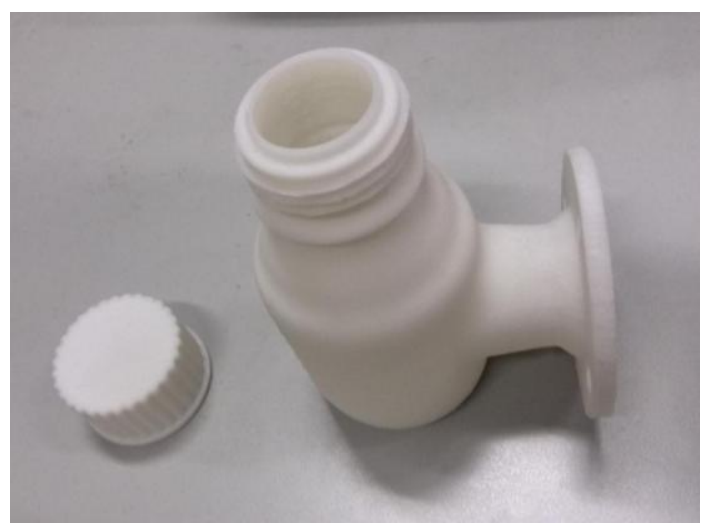

Figure 11. RP entity of MFC reactor

\section{Conclusions}

Reverse engineering software (Imageware) and forward engineering software (Solidworks) were combined to reverse reconstruct the 3D model of the MFC reactor with complex spatial structure, which greatly shorten the production cycle without the traditional engineering drawing.

The effective combination of RE and RP was in line with the requirements of MFC development, and provided reference for optimizing the MFC reactor.

\section{Acknowledgments}

This work was financially supported by a project funded by the Priority Academic Program Development of Jiangsu Higher Education Institutions (PAPD), Natural Science Foundation of Jiangsu Province (No. BK20161289), Applied Research Projects of Nantong City (GY12016054, GY12016056), Open Fund Project of Jiangsu Key Laboratory of 3D Printing Equipment and Application Technology (Nantong Institute of Technology) 
(2017KFKT02、2017KFKT09 、2017KFKT10), and Nantong Key Laboratory of 3D printing technology and Application (CP12016002).

\section{References}

1. L. Zhuo, X.X. Wang, S.S. Lv, M.Y. Huang, H.J. Ning, Mod Chem Ind 8, 41 (2017)

2. J. Ma, D.Y. Su, H.K. Pu, X.X. Wang, Q.Q.Chen, Chinese Journal of Power Sources 39 (10), 2318 (2015)

3. Y. Zhu, X.W. Li, J.D. Wei, X.X. Wang, Y.Y. Zhu, H.J. $\mathrm{Ni}$, Plastics Science and Technology 45 (4), 79 (2017)
4. X.X. Wang, J.D. Wei, Y. Pei, Y. Zhu, H.J. Ni, Appl. Mech. \& Mater. 752-753, 1301 (2015)

5. T. Rayna, L. Striukova. Technological Forecasting \& Social Change 102, 214 (2016)

6. D.T. Li, X.B. Feng, P. Liao, H.J. Ni, Y.D. Zhou, M.Y. Huang, Z.Y. Li, Y. Zhu, Lecture Notes in Electrical Engineering 269 , 1919 (2014)

7. C. J, C.S. Xiang, R.Z. Liu, Opto-Elec Eng 43 (7), 40 (2016)

8. V.N. Chougule,A.V. Mulay, B.B. Ahuja, Procedia Eng. 97, 212 (2014)

9. K. Bian, K.E. Yinglin, Chin. J. Mech. Eng. 26,197 (2013)

10. S.A. Khaled, J.C. Burley, M.R. Alexander, J. Yang, Int. J. Pharm 494 (2), 643 (2015) 